\title{
5
}

\section{Role of Progesterone Receptors in Mammary Development and Carcinogenesis}

\author{
Gopalan Shyamala
}

\section{Introduction}

It is well established that signaling through estrogen and progesterone through their cognate receptors, estrogen receptor $\alpha(\mathrm{ER} \alpha)$ and progesterone receptor (PR) are essential for both the development of mammary glands and their transformation to preneoplastic and neoplastic counterparts. Several years ago it was demonstrated that both mouse and human mammary epithelial cells proliferate in response to progesterone (1-3). Despite this, among the two ovarian steroids, estrogens have been traditionally associated with an increased risk for breast cancer. In recent years, several studies, including our own, provide compelling evidence that progesterone/PR signaling may play a crucial role in the transformation of mammary epithelial cells. The identity of a particular cell is established during development and conversion of a normal cell to its malignant counterpart is accompanied by alterations in certain fundamental mechanisms that define its identity. Accordingly, to identify the mechanisms that trigger carcinogenesis, it is essential to understand the processes that ensure normal development and maintenance of this phenotype. This chapter summarizes our studies on PR during normal mammary development and their relevance to carcinogenesis.

\section{Regulation of PR Expression and its Role During Mammary Development}

Mammary development occurs mostly in the post natal female and is discontinuous. The initial phase of development accompanies puberty, commonly referred to as ductal morphogenesis, requires signaling through estrogen/ER $\alpha$. The second phase of development accompanies pregnancy. It results in lobulo-alveolar development, and requires signaling through progesterone/PR. Similar to other estrogen target tissues, in the mammary gland, the expression of PR is regulated by estrogen (4), whereby it serves as a marker for both estrogen and progesterone action. Therefore, during various mammary gland physiological states, the PR expression patterns and their role need to be evaluated within the context of both estrogen and progesterone action. 
In the mammary gland of pre-pubertal mice, PR gene expression is associated with the end bud cells (5), the putative undifferentiated progenitors which give rise to ductal cells. Studies on PR-null mutant mice reveal that PR is not essential for the ductal growth accompanying puberty (6). Thus, the expression of $\mathrm{PR}$ in the end bud cells does not reflect its requirement for ductal morphogenesis, but is indicative of the estrogenic sensitivity of these cells. In the mammary gland of adult females, the PR steady-state levels are modulated as a function of estrous/menstrual cycle. Estrogen regulates its expression positively which, in turn, is counteracted by progesterone. In mammary ducts of adult females, PR expression is heterogeneous, and the percentage of PR positive cells does not change either after ovariactomy or exposure to estradiol, despite changes in the steady state levels of PR (7), indicating that its expression is restricted to a subset of epithelial cells. Since epithelial cell proliferation is promoted by signaling through PR, which gives rise to alveolar cells, it is likely that in the adult female, PR positive cells represent the putative progenitors of alveolar cells (6).

A paradox that emerges from studies on the developmental regulation of PR is that while its expression is essential for lobulo-alveolar development, it is down regulated during pregnancy and lactation. This down-regulation is evident both at the level of mRNA and protein (4), and is accompanied by a significant decrease in the number of PR positive epithelial cells (8). It is well known that, for the most part, cellular proliferation and differentiation represent two incompatible phenomena, and lobulo-alveolar development is indicative of both morphological and functional differentiation. Thus, one of the keys for switching the epithelial cells from a proliferative to differentiated state may be the loss of PR expression. As such, in instances where the mechanisms that regulate PR expression are deregulated, cell proliferation can proceed in an unabated manner and thus, trigger transformation. Indeed, this appears to be the case, as revealed by our studies using one of the transgenic models created by our laboratory and discussed below.

\section{Overexpression of the ' $A$ ' Form of PR (PR-A) Causes Transformation of Mammary Epithelial Cells}

It is well established that PR exists in two molecular forms commonly referred to as the ' $A$ ' and ' $B$ ' forms whose ratio varies among target tissues (9). In adult rodents, the mammary gland ratio of the 'A':' $B$ ' forms is $3: 1(10)$. In-vitro studies have shown that the ' $A$ ' and ' $B$ ' forms can have different functions in the same cell. In addition, the $P R$ activity of the individual form can vary among different types of cells (11). Furthermore, depending on the cell, the ' $A$ ' form can either inhibit or enhance the activity of the PR' $B$ ' form (11). Based on these observations, it is believed that appropriate cellular responsiveness to progesterone is dependent on regulated expression and/or activity of the two PR forms (12). Therefore, an aberration in normal mammary development may result from inappropriate progesterone signaling due to an imbalance in the expression and/or activities of the 
two PR forms. The imbalance, in turn, may have implications for mammary oncogenesis. To test this hypothesis, our laboratory created transgenic mice in which the native PR 'A':'B' ratio was altered by introducing additional PR ' $A$ ' or ' $B$ ' forms as transgene. A binary system was used for the expression of the transgene as shown in Figure 1. Detailed descriptions of the constructs have been published $(13,14)$.

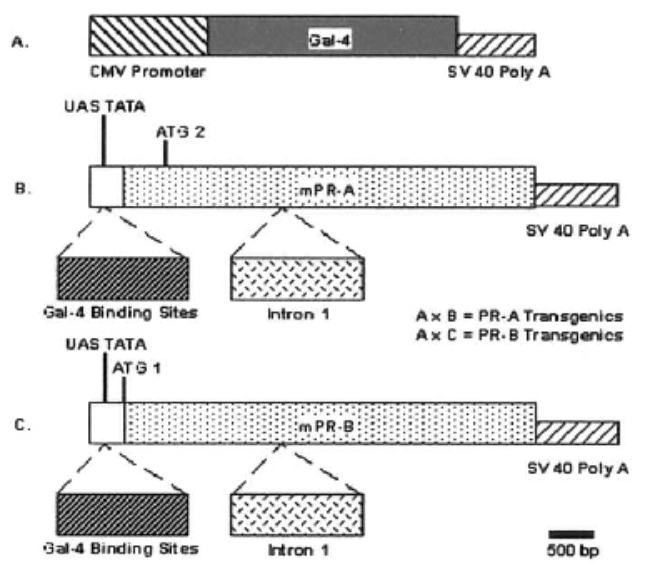

Figure 1. Schematic representation of plasmid construction for the binary system. (A) Insertion of the GAL-4 gene into the $\mathrm{CMV}$ promoter expression plasmid containing simian virus (SV) 40 splice and polyadenylylation sequences. (B) $\mathrm{mPR}$ cDNA (A form with only ATG 2) containing intron 1 and SV40 splice and polyadenylation sequences fused to UAS-TATA fragment containing four GAL-4 binding sites. E, EcoR1. (C) MPR cDNA (B form with only

ATG1) containing intron 1 and SV40 splice and polyadenylation sequences fused to UAS-TATA fragment containing four GAL-4 binding sites.

Mammary development is abnormal in transgenic mice carrying an imbalance in the native ratio of ' $A$ ':' $B$ ' forms by overexpressing either the ' $A$ ' or ' $B$ ' form (referred to as PR-A and PR-B transgenics, respectively) $(13,14)$. In particular, PR-A transgenic mammary glands exhibit excessive ductal growth, and morphological and histological features associated with transformed cells, i.e., loss in basement membrane integrity and cell-cell adhesion. The aberrant patterns of ductal growth were not restricted to the offspring of any particular founder mouse, establishing that the phenotype was due to the transgene expression, and not due to their integration into any unique site on the genome (15).

In a series of comprehensive studies, Medina, et al. identified certain molecular markers unique to mammary epithelial cells at various stages of transformation. In particular, they showed that in mouse mammary epithelial cells, a decrease in p21 expression, without an increase in cyclin D1, is indicative of immortalization, and precedes the onset of hyperplasia/preneoplasia (16). Among the characteristics that distinguish the presumptive immortalized epithelial cells from those in hyperplasia/preneoplasia are increases in cyclin D1 expression and cell proliferation, and a decrease in ER $\alpha$ expression (17). 


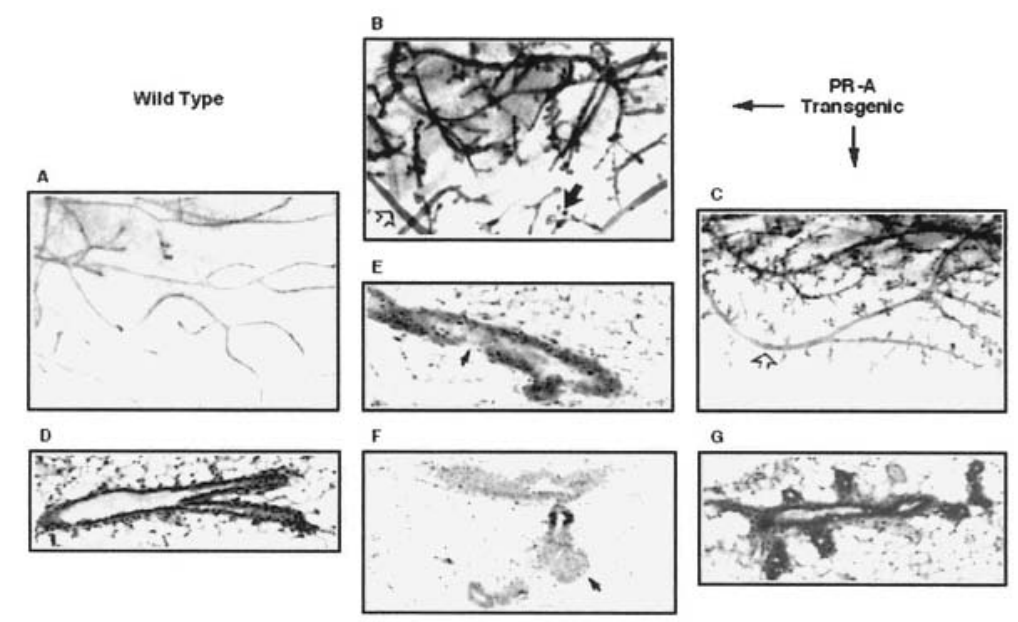

Figure 2. Morphological and histological characteristics of wild type and PR-A in adult transgenic mice. Whole-mounts (A-C) and histology (D-G) of mammary glands from adult (10-14 weeks old) control PR-A transgene-negative (A and D) and PR-A transgenic mice (B, C, and E-G) are shown. In B and C, open arrows point to thick ducts, while solid arrows point to clustered buds at the tip of ducts. Note that, in contrast to the monolayer associated with the duct from wild type mice (D), the ducts in PR-A transgenics are composed of multilayered cells ( $\mathbf{E}, \mathbf{F}$, and G). In $\mathrm{E}$, the arrow points to an indistinct epithelial-stromal boundary, and in $\mathrm{F}$, the arrow points to disorganized masses of cells at the tip of a duct.

In mammary glands of PR-A transgenics, ducts with normal histology, displayed a decrease in $\mathrm{p} 21$ expression (Figure $3 \mathrm{~A}$ ) without an elevation in cyclin D1 (Figure 3B), or cell proliferation (Figure 3C). Similar to ducts with normal histology, in mammary dysplasias of PR-A transgenic, there was a decrease in $\mathrm{p} 21$ expression (Figure 3A), but was accompanied by an increase in cyclin D1 expression (Figure 3B) and cell proliferation (Figure 3C), and a decrease in ER $\alpha$ expression (Figure 3D). Therefore, these studies establish that mammary glands of PR-A transgenics contain at least two distinct populations of transformed epithelial cells: one, corresponding to immortalized cells, in early stages of transformation, present in the ducts with normal histology; and two, cells in later stages of transformation associated with dysplasias.

An imbalance in the native ratio of PR A:B isoforms also exists in PR-B transgenic mice, but the mammary phenotype of these mice is distinct from that of the PR-A transgenics (14). In fact, PR-B mammary glands have impaired ductal elongation. Analyses of these mammary glands did not reveal any detectable changes in either the number of BrdU-positive cells or the expression of cyclin D1, $\mathrm{p} 21$, or $\operatorname{ER} \alpha(18)$. 

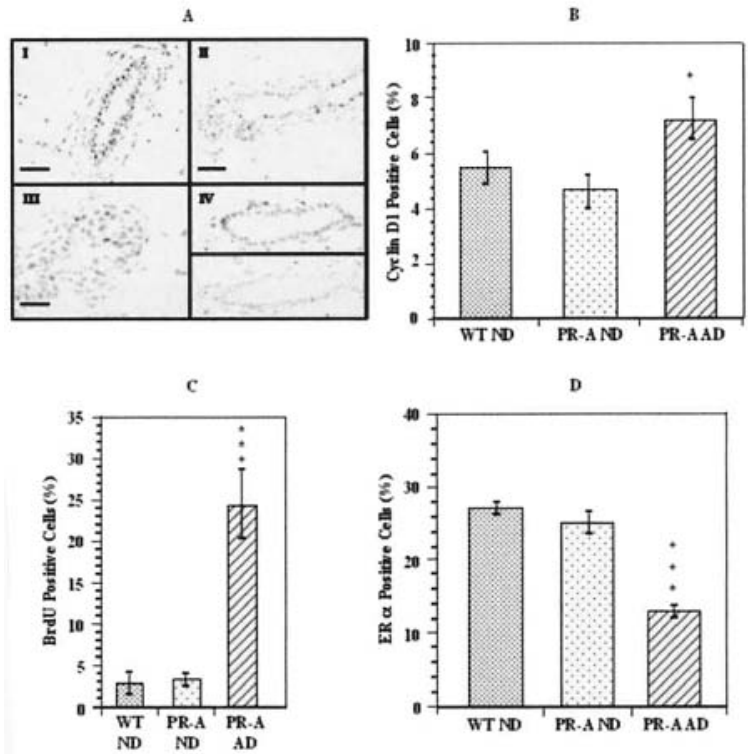

$20 \mu \mathrm{m}$. Panel B shows the number of cyclin D1-positive cells in normal (ND) and abnormal ducts (AD). Panel $\mathbf{C}$ shows the rate of cell proliferation, using BrdUpositive cells as index, in ND and AD. Panel D shows the number of ER $\alpha$-positive epithelial cells in ND and AD.

\section{Aberrant Features of Mammary Dysplasias in PR-A Transgenic Mice are Unaffected by Mifepristone}

The presence of at least two distinct populations of transformed epithelial cells, in mammary glands of PR-A transgenics, was also apparent from their sensitivity to the antiprogestin, mifepristone. While mifepristone abolished BrdU immunostaining in the ducts displaying normal histology, and in those of wild type mice, it did not alter the number of BrdU-positive cells in dysplastic lesions (18). Similarly, in these lesions, mifepristone did not effect the expression of either cyclin D1 or ER $\alpha$. Thus, it appears that deregulation of progesterone action, resulting from an overexpression of PR-A form, may not only trigger transformation of mammary epithelial cells, but may also dictate their phenotype in later stages of transformation.

\section{Progesterone/PR Signaling in Mammary Carcinogenesis}

Most of the classical studies on the developmental biology of the normal mammary gland and mammary oncogenesis have been done in rodents. Conclusions derived from these studies have been found to be applicable to the human condition (19). Furthermore, the pattern of PR localization in normal mouse mammary glands is 
similar to that reported previously for human breast, i.e., (a) PR expression in the epithelial cells is heterogeneous, and (b) the connective tissue surrounding these epithelial cells are PR free $(7,20)$.

In normal mammary epithelial cells, the initial impact of progesterone signaling through PR results in an increase in proliferation. Therefore, in its capacity as a mammary gland mitogen, progesterone has the potential to trigger carcinogenesis. An excellent example of this $\mathbf{P}$ action is the animal model developed by Lanari et al. in which the progestin, medroxyprogesterone induces mammary tumors (21). Another example is the p53-null mutant mammary epithelium in which progesterone alone strongly enhances mammary tumorigenesis (22). Studies from our laboratory (as reviewed here) showed that a deregulation in progesterone action (as in PR-A transgenics) can alter the growth potential of epithelial cells, at least, in part, at the level of cell cycle, leading to transformation. Recent population based studies also demonstrated that, in uninterrupted combined hormone (estrogen + progestin) replacement therapy (CHRT), progesterone is the contributing factor for the increased risk for mammary carcinogenesis (23). Also, studies on normal mammary development established that the net outcome of signaling through PR is to drive the epithelial cells towards lobulo-alveolar development. Thus, it is noteworthy that women who are at increased risk for mammary oncogenesis due to CHRT develop lobular carcinomas (24).

Studies from our laboratory clearly established that an imbalance in the expression of the two PR isoforms, resulting from PR-A overexpression, may lead to transformation of mammary epithelial cells. An imbalance in the relative ratio of PR A:B isoforms has also been observed in certain human mammary tumors, and has often been associated with overexpression of the PR-A form (25-27).

\section{Concluding Remarks}

Our studies on the role of progesterone/PR in the mammary gland highlight their involvement in mammary epithelial cell proliferation during development. In addition, they establish that appropriate expression of the two PR isoforms is critical for normal mammary development. Finally, they demonstrate that an imbalance in the native ratio of the two isoforms, resulting in dominance of the PRA form, can trigger transformation. Thus, PR-A transgenic mice can serve as an important experimental model for dissecting the mechanisms underlying progesterone-dependent mammary epithelial cell transformation, and progression in vivo. In addition, they may help to devise strategies for arresting the progression of transformation, and reversing the transformed phenotype.

\section{Dedication}

This article is dedicated to the memory of my dear friend Jane Toft, who lived a life of a scientist/teacher and wife/mother with courage, honesty, and devotion. 


\section{Acknowledgement}

These studies were supported by NIH Grant CA 66541 .

\section{References}

1. Bresciani F (1968) Topography of DNA synthesis in the mammary gland of the $\mathrm{C} 3 \mathrm{H}$ mouse and its control by ovarian hormones: an autoradiographic study. Cell Tissue Kinetics 1:51.

2. Ferguson DJ, Anderson TJ (1981) Morphological evaluation of cell turnover in relation to the menstrual cycle in the "resting" human breast. Br J Cancer 44:177-181.

3. Going JJ, Anderson TJ, Battersby S, et al (1988) Proliferative and secretory activity in human breast during natural and artificial menstrual cycles. Amer J Pathol 130:193-204.

4. Shyamala G, Schneider W, Schott D (1990) Developmental regulation of murine mammary progesterone receptor gene expression. Endocrinology 126:2882-2889.

5. Silberstein GB, Van Horn K, Shyamala G, et al (1996) Progesterone receptors in the mouse mammary duct: distribution and developmental regulation. Cell Growth Differ 7:945-952.

6. Shyamala G (1999) Progesterone signaling and mammary gland morphogenesis. J Mammary Gland Biol Neoplasia 4:89-104.

7. Shyamala G, Barcellos-Hoff MH, Toft D, et al (1997) In situ localization of progesterone receptors in normal mouse mammary glands: absence of receptors in the connective and adipose stroma and a heterogeneous distribution in the epithelium. J Steroid Biochem Mol Biol 63:251-259.

8. Shyamala G, Chou YC, Louie SG, et al (2002) Cellular expression of estrogen and progesterone receptors in mammary glands: regulation by hormones, development and aging. J Steroid Biochem Mol Biol 80:137-148.

9. Tsai MJ, O'Malley BW (1994) Molecular mechanisms of action of steroid/thyroid receptor superfamily members. Ann Rev Biochem 63:451-486.

10. Schneider W, Ramachandran C, Satyaswaroop PG, et al (1991) Murine progesterone receptor exists predominantly as the 83-kilodalton ' $\mathrm{A}$ ' form. $\mathrm{J}$ Steroid Biochem Mol Biol 38:285-291.

11. Vegeto E, Shahbaz MM, Wen DX, et al (1993) Human progesterone receptor A form is a cell- and promoter-specific repressor of human progesterone receptor B function. Mol Endocrinol 7:1244-1255.

12. McDonnell DP (1995) Unraveling the human progesterone receptor signal transduction pathway: Insights into antiprogestin action. Trends Endocrinol Metabol 6:133-138. 
13. Shyamala G, Yang X, Silberstein G, et al (1998) Transgenic mice carrying an imbalance in the native ratio of $A$ to $B$ forms of progesterone receptor exhibit developmental abnormalities in mammary glands. Proc Natl Acad Sci USA 95:696-701.

14. Shyamala G, Yang X, Cardiff RD, et al (2000) Impact of progesterone receptor on cell-fate decisions during mammary gland development. Proc Natl Acad Sci USA 97:3044-3049.

15. Shyamala G, Louie SG, Camarillo IG, et al (1999) The progesterone receptor and its isoforms in mammary development. Mol Genet Metab 68:182-190.

16. Said TK, Moraes RC, Singh U, et al (2001) Cyclin-dependent kinase (cdk) inhibitors/cdk4/cdk2 complexes in early stages of mouse mammary preneoplasia. Cell Growth Differ 12:285-295.

17. Medina D (2002) Biological and molecular characteristics of the premalignant mouse mammary gland. Biochim Biophys Acta 1603:1-9.

18. Chou YC, Uehara N, Lowry JR, et al (2003) Mammary epithelial cells of PR-A transgenic mice exhibit distinct alterations in gene expression and growth potential associated with transformation. Carcinogenesis 24:403-409.

19. Cardiff RD, Wellings SR (1999) The comparative pathalogy of human and mouse mammary glands. J Mammary Gland Biol Neoplasia 4:105-122.

20. Press MF, Greene GL (1988) Localization of progesterone receptor with monoclonal antibodies to the human progestin receptor. Endocrinology 122:1165-1175.

21. Lanari C, Molinolo AA (2002) Progesterone receptors-animal models and cell signalling in breast cancer. Diverse activation pathways for the progesterone receptor: possible implications for breast biology and cancer. Breast Cancer Res 4:240-243.

22. Medina D, Kittrell FS, Shepard A, et al (2003) Hormone dependence in premalignant mammary progression. Cancer Res 63:1067-1072.

23. Weiss LK, Burkman RT, Cushing-Haugen KL, et al (2002) Hormone replacement therapy regimens and breast cancer risk(1). Obstet Gynecol 100:1148-1158.

24. Daling JR, Malone KE, Doody DR, et al (2002) Relation of regimens of combined hormone replacement therapy to lobular, ductal, and other histologic types of breast carcinoma. Cancer 95:2455-2464.

25. Graham JD, Yeates C, Balleine RL, et al (1995) Characterization of progesterone receptor $\mathrm{A}$ and $\mathrm{B}$ expression in human breast cancer. Cancer Res 55:5063-5068.

26. Mote PA, Johnston JF, Manninen T, et al (2001) Detection of progesterone receptor forms $\mathrm{A}$ and $\mathrm{B}$ by immunohistochemical analysis. J Clin Pathol 54:624-630.

27. Mote PA, Bartow S, Tran N, et al (2002) Loss of co-ordinate expression of progesterone receptors $A$ and $B$ is an early event in breast carcinogenesis. Breast Cancer Res Treat 72:163-172. 